\title{
Negative differential resistance due to single-electron switching
}

\author{
C. P. Heij, D. C. Dixon, P. Hadley, J. E. Mooij \\ Applied Physics and DIMES, Delft University of Technology \\ Lorentzweg 1, 2628 CJ Delft, The Netherlands
}

(October 9, 2018)

\begin{abstract}
We present the multilevel fabrication and measurement of a Coulomb-blockade device displaying tunable negative differential resistance (NDR). Applications for devices displaying NDR include amplification, logic, and memory circuits. Our device consists of two $\mathrm{Al} / \mathrm{Al}_{x} \mathrm{O}_{y}$ islands that are strongly coupled by an overlap capacitor. Our measurements agree excellently with a model based on the orthodox theory of single-electron transport.
\end{abstract}

Single-electron tunneling devices offer a means to manipulate individual electrons. Their advantages of small size and low power dissipation have stimulated a number of proposals for their use in some future generation of computation technology 1 yet relatively few such circuits have been measured. Many techniques exist for creating single-electron devices, including the use of scanning probes to manipulate the nanometer scale structures necessary for room temperature operation. These structures have so far been limited to planar layouts, which severely restrict possible circuit architectures because voltage gain becomes difficult to achieve and wire crossing is impossible. The most widely used fabrication technique, electron beam lithography, allows one to build multilayered circuits that can circumvent these problems. E-beam lithography, however, has a minimum resolution of $\sim 10 \mathrm{~nm}$; consequently, single-electron effects can usually only be observed at low temperature $(<1 \mathrm{~K})$ in devices built this way. Up to now, technologically oriented research has primarily focused on the further miniaturization of basic components, while ignoring the fabrication and testing of more complex circuits. We feel it is worthwhile to consider low-temperature prototypes of these circuits to estimate the usefulness of their future high-temperature counterparts.

In this Letter we report on a multilayer circuit consisting of two strongly coupled $\mathrm{Al} / \mathrm{Al}_{x} \mathrm{O}_{y}$ islands fabricated using electron beam lithography and measured at low temperature. The circuit demonstrates negative differential resistance (NDR) due to the tunneling of a single electron into one of the islands. Device applications of NDR - including amplification, logic and memory - have been extensively discussed in literature regarding resonant tunnel diodes. 1 Single-electron NDR has heretofore been predicted in systems of multiple islands, where electrostatic repulsion between electrons in different islands regulates the source-drain current 3 Our circuit offers the advantage of requiring only two islands, rather than six arranged in a zig-zag,

The equivalent circuit diagram of our device is shown in Fig. 1a. The left island forms a single-electron transistor (SET), allowing a current $I$ to flow between the voltage source $V_{b}$ and ground. The right island, how- ever, merely traps charge entering from the source, and so acts as an "electron box." coupled by a large mutual capacitance $C_{m}$, but electron tunneling between the islands is forbidden. Additional control is provided by tuning gate voltages $V_{g 1}$ and $V_{g 2}$, which determine the electrostatic potentials and charge states of the islands. The current through a solitary SET depends both upon the bias voltage across its terminals and the gate voltage. In our setup the SET feels an additional effective gate voltage due to the charge state of the electron box. Whenever a single extra electron tunnels into the box, there is a discontinuous change in charge on $C_{m}$, resulting in a jump in the effective gate voltage felt by the SET and consequently a jump in the current.
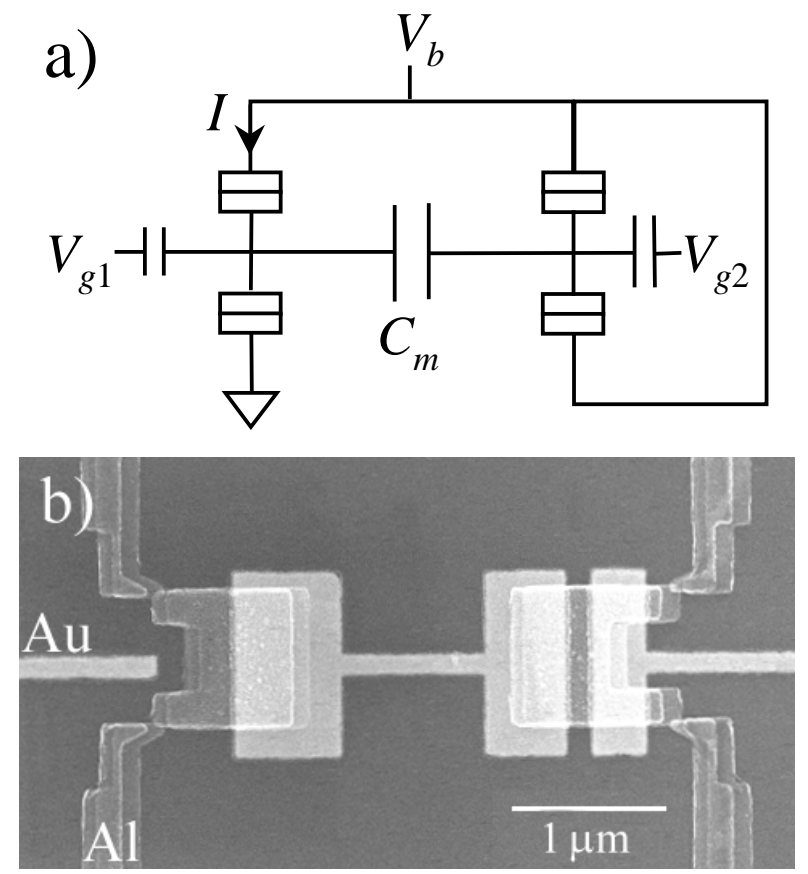

FIG. 1. a) Schematic circuit diagram of the device. Electrons flow from source to drain through the left island, while the right island forms an "electron box." b) SEM photograph of the multilevel device, with two $\mathrm{Al} / \mathrm{Al}_{x} \mathrm{O}_{y}$ islands coupled to each other by an underlying Au layer. The islands' potentials can also be tuned using gate electrodes in this layer. 
The NDR mechanism is as follows. The SET is tuned so that it conducts at low $V_{b}$, while the box is in Coulomb blockade. When $V_{b}$ is increased past some threshold voltage and overcomes this blockade, an electron tunnels into the box and becomes trapped. Due to the mutual capacitance $C_{m}$, this extra electron increases the electrochemical potential of the SET and pushes it into Coulomb blockade, decreasing $I$. In other words, the addition of a single extra electron to the box switches the SET from "on" to "off." Simulations (described below) have shown that $C_{m}$ should be approximately larger than the junction capacitance in order to see the effect. Junction capacitances $C_{j}$ of $0.2-0.3 \mathrm{fF}$ are possible in $\mathrm{Al} / \mathrm{Al}_{x} \mathrm{O}_{y}$, the material of choice for most single-electronics experiments. Such a high value of $C_{m}$ is difficult to achieve in a planar, single-layer design 6 Much higher capacitances are attainable by overlappinf circuit elements, which requires multilevel fabrication

Figure 1b shows an SEM photograph of our device, consisting of an Al island layer (medium gray) and an underlying $\mathrm{Au}$ gate layer (light gray), with a thin intermediate $\mathrm{SiO}$ layer providing electrical insulation. The tunnel junctions are formed at the corners of the islands where they meet the pointed ends of the leads. Three sections of the gate layer are visible; the two structures extending from the left and right sides are the tuning gates, while the central dumbbell-shaped structure underlapping both islands forms the mutual capacitor $C_{m}$.

The device was fabricated on a silicon substrate with a $250 \mathrm{~nm}$ thermally oxidized top layer, and patterned using standard electron beam lithography with a high resolution pattern generator in a double layer PMMA resist. The bottom gate layer was formed by evaporating $5 \mathrm{~nm}$ of $\mathrm{Ti}$ and $20 \mathrm{~nm}$ of $\mathrm{Au}$ perpendicular to the substrate surface. Directly after lift-off the whole sample was covered with a $32 \mathrm{~nm}$ insulating $\mathrm{SiO}$ layer. To ensure good step coverage, $\mathrm{SiO}$ was evaporated under four perpendicular angles oriented $30^{\circ}$ to the substrate surface normal. The islands, leads and contact pads were written in a new bilayer of PMMA after aligning the electron beam pattern generator to Au markers defined in the gate layer. A pattern generator alignment resolution of $50 \mathrm{~nm}$ or less is necessary to produce good results. The tunnel junctions were formed using the standard technique of double angle shadow evaporation of $\mathrm{Al}$ through the resist mask, oxidizing the $\mathrm{Al}$ between evaporations $\mathrm{O}$ Contact pads were coupled to the gates by $0.2 \mathrm{pF}$ overlap capacitors. To protect the junctions from high voltage static discharges, the leads were shunted on-chip by $12 \mathrm{pF}$ overlap capacitors.

The device was measured in a standard ${ }^{3} \mathrm{He}-{ }^{4} \mathrm{He}$ dilution refrigerator at a base temperature of $4 \mathrm{mK}$ (electron temperature $\approx 27 \mathrm{mK}$ ). An external magnetic field of 1 $\mathrm{T}$ was applied to suppress superconductivity. From highbias measurements, the total tunneling resistances of the SET and the box were determined to be $7 \mathrm{M} \Omega$ and 13 $\mathrm{M} \Omega$, respectively. Having verified that all the junctions had finite tunneling resistances, the leads were connected

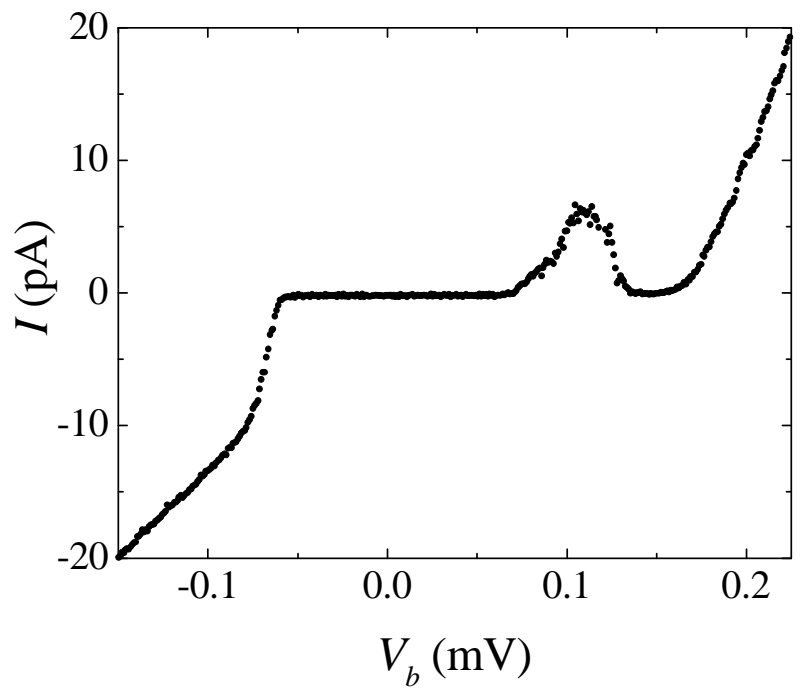

FIG. 2. Typical data sweep showing both NDR for positive bias and enhanced differential conductance for negative bias, both due to single-electron switching in the "electron box" island. The electron temperature is $27 \mathrm{mK}$.

as in Fig. 1a. No leakage current through the SiO insulating layer was detected.

A typical measurement of the current $I$ as a function of source-drain bias $V_{b}$ is plotted in Fig. 2. The device clearly demonstrates NDR in the bias range of $110-130 \mu \mathrm{V}$, with a peak-to-valley ratio in excess of 30 . A more precise determination of this ratio is difficult, however, due to an enhancement of low-frequency noise around the NDR onset, possibly caused by thermally activated charge fluctuations in the box. Fig. 2 also shows enhanced differential conductance at $V_{b} \approx-70 \mu \mathrm{V}$. This feature is due to a mechanism similar to NDR, but where the trapping of an extra electron in the box suddenly pulls the SET out of Coulomb blockade.

The NDR features can be shifted by tuning the gate voltages. The dependence on $V_{g 1}$ is shown in Fig. 3a, where the differential conductance $\partial I / \partial V_{b}$ is plotted in gray-scale as a function of $V_{b}$ and $V_{g 1}$. Here we see diamond-shaped Coulomb blockaded regions (marked by "I $=0 "$ ), fractured by the discrete charging of the box. Black regions represent NDR, while white represents enhanced differential conductance.

The electrostatic potentials of a two-island circuit may be expressed analytically in terms of the applied voltages the charge state of each island, and the capacitances. 9 Consequently, the slopes of the various thresholds in Fig. 3a, combined with similar measurements (such as by sweeping $V_{g 2}$ ), allow us to fully characterize the capacitor network of the device. The junction capacitances were all approximately $0.3 \mathrm{fF}$, while $C_{m}$ was estimated to be 0.64 $\mathrm{fF}$. Using our estimated capacitances, we have carried out simulations of the device using a master equation approach combined the orthodox theory of singleelectron tunneling 10,11 The simulation of Fig. 3b shows 
$\partial I / \partial V_{b}$ as a function of $V_{b}$ and $V_{g 1}$, assuming a temperature of $25 \mathrm{mK}$. It correctly reproduces the position and character of the features in Fig. 3a, with only minor variations. We similarly found excellent agreement between experiment and simulation when $V_{g 2}$ was swept.

Simulations show that the magnitude of NDR gradually decreases with increasing temperature, vanishing when:

$$
k T \gtrsim \frac{1}{4} \frac{e^{2} C_{m}}{C_{1} C_{2}-C_{m}^{2}}
$$

Here $C_{1(2)}$ is the total capacitance of the SET (box) including the coupling capacitance $C_{m}$. This maximum temperature was approximately $150 \mathrm{mK}$ for our device, and measurements at $T=100 \mathrm{mK}$ confirmed that NDR exists, but is greatly diminished, at this higher temperature. Simulations also predict that, for sufficiently large $C_{m}>3 C_{j}$, multiple NDR regimes should appear. We estimate that a fully optimized device using our multilevel

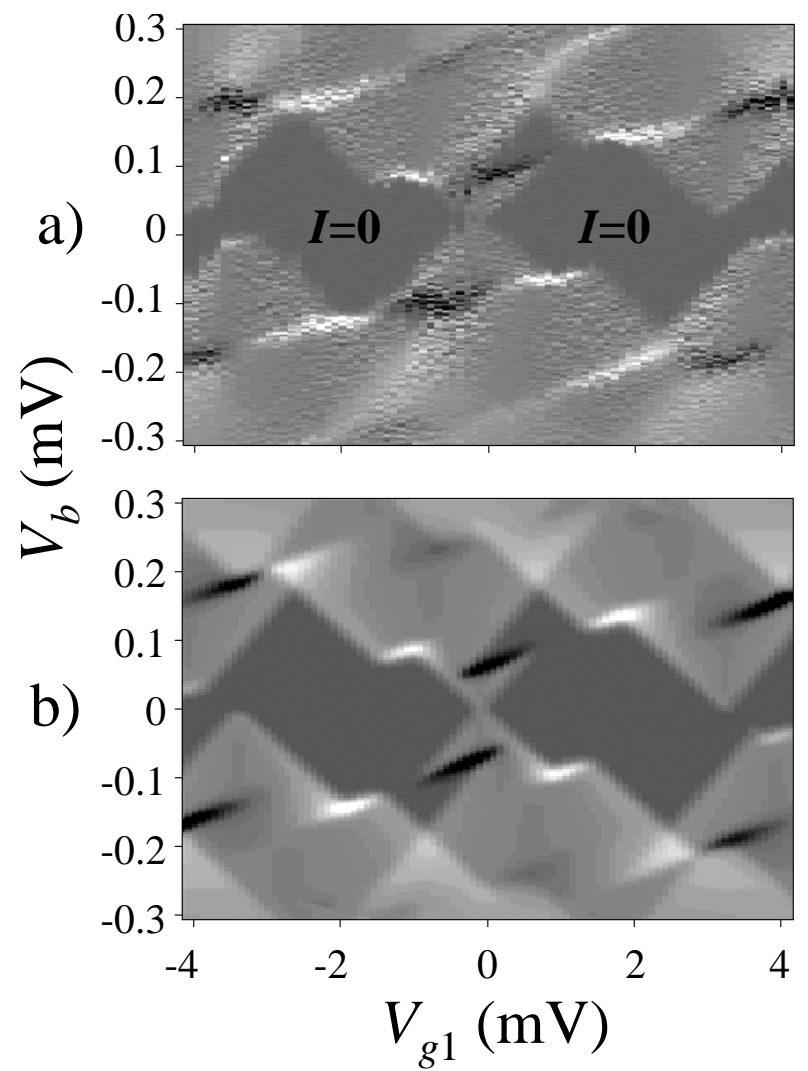

FIG. 3. a) Grayscale plot of $\partial I / \partial V_{b}$ as a function of $V_{g 1}$ and $V_{b}$, showing the tunability of the NDR onset (black). The periodicity of the SET is $3.5 \mathrm{mV}$. b) Master-equation simulation of the device, using capacitances estimated from multivariable fitting of the features in (a). technique could yield a $C_{m} / C_{j}$ ratio of 10 , making possible the study of very strongly coupled metallic islands.

In conclusion, we have measured negative differential resistance due to single-electron switching in a circuit with a strong capacitive coupling between two islands. Measurements were in excellent quantitative agreement with semiclassical simulations. Our multilevel fabrication process allows inter-island couplings unmatched by any planar architecture, perhaps allowing the observation of new physical effects. Preliminary measurements have produced encouraging results, and more research in this regime is forthcoming. Our measurements also demonstrate the strong influence that the introduction of a single electron can have on the conductance of a small island of charge - an effect that will only gain importance as the present trend of transistor miniaturization proceeds apace.

We acknowledge useful input from Paul McEuen, K. K. Likharev and Caspar van der Wal. This research was supported by CHARGE, Esprit project 22953, and by Stichting voor Fundamenteel Onderzoek der Materie (FOM).

${ }^{1}$ For a review, see A. N. Korotkov, in Molecular Electronics, ed. by J. Jortner and M. A. Ratner. Oxford: Blackwell, 1997.

2 Tunnel-Diode and Semiconductor Circuits, ed. by John M. Carroll. New York: McGraw-Hill, 1963.

${ }^{3}$ Hiroshi Nakashima and Kiyohiko Uozumi, Jpn. J. Appl. Phys. 34, L1659 (1995); ibid., Int. J. Electronics 83, 333 (1997).

${ }^{4}$ Mincheol Shin, Seongjae Lee, Kyoung Wan Park, and ElHang Lee, Phys. Rev. Lett. 80, 5774 (1998).

${ }^{5}$ P. Lafarge, H. Pothier, E. R. Williams, D. Esteve, C. Urbina and M. H. Devoret, Z. Phys. B 85, 327 (1991).

${ }^{6}$ G. Zimmerli, R. L. Kautz, and John M. Martinis, Appl. Phys. Lett. 61, 2616 (1992).

${ }^{7}$ E. H. Visscher, S. M. Verbrugh, J. Lindeman, P. Hadley, and J. E. Mooij, Appl. Phys. Lett. 66, 305 (1995).

${ }^{8}$ T. A. Fulton and G. J. Dolan, Phys. Rev. Lett. 59, 109 (1987).

9 See, for example, David C. Dixon, Ph.D. thesis, University of California (1998).

${ }^{10}$ D. V. Averin and K. K. Likharev, in Mesoscopic Phenomena in Solids, eds. B. L. Altshuler, P. A. Lee, and R. A. Webb, Elsevier (1991).

${ }^{11}$ G.-L. Ingold and Yu. V. Nazarov, in Single Charge Tunneling, ed. by H. Grabert and M. H. Devoret, New York: Plenum, 1992, pp.21-108. 